\title{
riccafd
}

Revista Iberoamericana de Ciencias de la Actividad Física y el Deporte

\section{EDUCAJUDO: INNOVACIÓN EDUCATIVA Y DE SALUD EN MAYORES. APRENDIENDO A CAERSE (YOKO-UKEMI)}

EDUCAJUDO: EDUCATIONAL AND HEALTH INNOVATION FOR THE ELDERLY. LEARNING TO FALL (YOKO-UKEMI)

Campos Mesa, $\mathrm{MC}^{1}$; DelCastillo-Andrés, $\mathrm{O}^{2}$; Castañeda Vázquez, $\mathrm{C}^{3}$ y Toronjo Hornillo, $\mathrm{L}^{4}$.

1 Campos Mesa, MC., Profesora Ayudante Doctor en el Departamento de Educación Física y Deporte. Grupo de Investigación HUM-507. Universidad de Sevilla. España (mccampos@us.es)

2 DelCastillo-Andrés, O., Profesor titular de Educación Física E.S.O. Doctor en Ciencias de la Educación. Departamento de Educación Física y Deporte. Grupo de Investigación HUM-507. Universidad de Sevilla. España (ocastillo@us.es)

3 Castañeda Vázquez, C., Profesora Ayudante Doctor en el Departamento de Educación Física y Deporte. Grupo de Investigación HUM-507. Universidad de Sevilla. España (carolinacv@us.es)

4 Toronjo Hornillo, L., Licenciado en Ciencias de la Actividad Física y del Deporte. Grupo de Investigación HUM-507. Universidad de Sevilla. España (Itoronjo@gmail.com)

Código UNESCO: 5801 Teoría y métodos educativos

Clasificación Consejo de Europa: 5 Actividad Física y Salud

Recibido el 24 de junio de 2015

Aceptado el 18 de septiembre de 2015

Correspondencia:

Campos Mesa, MC.: mccampos@us.es

\section{RESUMEN}

El envejecimiento de la población española es una realidad incipiente debido al aumento de la esperanza de vida. Sin embargo, el proceso de envejecimiento puede convertir a las personas mayores en frágiles y dependientes. Una de las causas de fragilidad es el alto número de caídas que sufren las personas mayores y las consecuencias que conllevan. El objetivo del presente trabajo es presentar un diseño de intervención para aplicar el Judo en esta población 
como elemento educativo en la enseñanza del control de la caída, para minimizar los daños producidos por el impacto del cuerpo contra el suelo. La intervención va dirigida a sujetos sanos y prefrágiles de cualquier sexo. Para llevar a cabo la enseñanza de las caídas en las personas mayores se plantean dos secuencias de aprendizaje: una asistida autónoma con implementos y otra asistida colaborativa con compañero (con o sin implementos).

PALABRAS CLAVES: fragilidad, judo, adultos-mayores, caídas, innovación educacional.

\section{ABSTRACT}

The aging of the Spanish population is an emerging reality due to an increase in life expectancy. However, the aging process can make the elderly frail and dependent. One of the causes of fragility is the high number of falls suffered by seniors and their implications. Therefore, the objective of this communication is to present an intervention design to apply Judo in this population as an educational tool in teaching fall control to minimize damage from the impact of the body against the floor. The intervention is aimed at healthy subjects and pre-fragile of either sex. To carry out the teaching of falls in older people two learning sequences are proposed: autonomous assisted with equipment and assisted with other collaborative partner (with or without equipment).

KEY WORDS: fragility, judo, adults-elderly, falls, educational innovations.

\section{INTRODUCCIÓN}

El envejecimiento de la población española es una realidad incipiente. Según el Instituto Nacional de Estadística ${ }^{1}$, este envejecimiento, acelerado por el aumento de la esperanza de vida, nos llevará a una población concentrada en la mitad superior de la pirámide de población, creciendo los grupos de edad a partir de los 50 años. En este sentido, España presenta la esperanza de vida 
más alta de Europa ${ }^{2}$. Sin embargo, a medida que aumenta dicha esperanza, el proceso de envejecimiento tiende a quebrar la salud de las personas mayores y hacerla más frágil ${ }^{3}$. Según el Ministerio de Sanidad, Servicios Sociales e Igualdad, "(...) la fragilidad es un estado de pre-discapacidad, de riesgo de desarrollar nueva discapacidad desde una situación de limitación funcional incipiente, y su importancia es que se centra en la funcionalidad y no focaliza en el diagnóstico de enfermedad"4. En base a la anterior definición, la fragilidad es un buen predictor de eventos adversos de salud a corto, medio y largo plazo, como puede ser, tener una caída.

Las intervenciones centradas en la actividad física han demostrado su eficacia en retrasar e, incluso, revertir la fragilidad y la discapacidad ${ }^{5}$. Pero a pesar de estas intervenciones sobre la condición física, los mayores están sometidos al riesgo de caerse. Según los datos facilitados por el Ministerio de Sanidad, en España las personas mayores de 65 años, sufren al menos una caída al año, tasa que al llegar a los mayores de 84 se dispara, multiplicándose por ocho ${ }^{6}$. En esta misma línea, la Organización Mundial de la Salud alertaba de la incidencia de las caídas como la segunda causa de fallecimientos por lesiones accidentales y explicita el hacer especial hincapié en la educación de los mayores y su capacitación, priorizando la investigación relacionada con las caídas ${ }^{7}$.

En relación a lo expuesto anteriormente, el Judo, como actividad física fundamentada en técnicas específicas sobre las caídas (ukemis), se convierte en una herramienta de gran valor que nos puede permitir reducir la fragilidad de las personas mayores actuando sobre su principal factor de riesgo, la caída. Las características de las propias técnicas del Judo $^{8}$ y la reducción de la magnitud de los impactos que se generan sobre el organismo aplicando estas técnicas ${ }^{9,10}$, son algunos de los factores de mayor interés para disminuir el riesgo de lesión en el caso de las personas mayores. 
Pensamos que nuestra intervención educativa es muy novedosa y tendrá una repercusión muy positiva. Tras una profunda revisión bibliográfica en las principales bases de datos científicas, no se han encontrado evidencias de intervenciones de este tipo donde se aplican los ukemis a través de una adaptación de las técnicas de enseñanza de la caídas de Judo, posibilitando su inclusión en las sesiones de acondicionamiento físico dirigidas a las personas mayores, contribuyendo a disminuir el miedo a caer, enseñándoles las técnicas que permitan reducir las consecuencias de las caídas.

Por tanto, el objetivo fundamental de este trabajo se centra en aplicar el Judo en personas mayores como elemento educativo en la enseñanza del control de la caída para minimizar los daños producidos por el impacto del cuerpo contra el suelo.

\section{MATERIAL Y MÉTODO}

\section{Muestra}

Para la selección de la muestra nos centraremos en recientes investigaciones $^{11,12}$ que, según constructo ${ }^{3}$, establecen una escala Rasgo de fragilidad para establecer, si el adulto-mayor es sano, prefrágil o frágil. En relación a los parámetros que establecen estos autores, nuestra propuesta de intervención va dirigida a una muestra clasificada como sana y prefrágil ${ }^{11}$, dentro de los parámetros de fragilidad que se establecen sobre la población adulta. Los criterios de inclusión para realizar esta propuesta son: personas mayores de cualquier sexo que no tengan diagnosticadas ninguna enfermedad que les impida realizar los ejercicios propuestos y tener movilidad en los segmentos corporales. Así mismo, deben firmar su consentimiento para la intervención. La captación de la muestra se realizará a través de centros y/o asociaciones que oferten actividad física para personas mayores. 


\section{Procedimiento}

Nuestra línea de intervención se basa en contenidos técnicos del Judo tradicional, o fundamentos del Judo Kodokan, creado en Japón en el año 1882 por el Maestro Jigoro Kano. Las características de las propias técnicas del Judo $^{8}$ y la reducción de la magnitud de los impactos que se generan sobre el organismo aplicando estas técnicas ${ }^{10}$, son algunos de los factores de mayor interés para disminuir el riesgo de lesión en el caso de caída de las personas mayores.

Centrándonos en los distintos tipos de ukemis ${ }^{13}$ vamos a desarrollar un trabajo colaborativo sobre Yoko-Ukemi (caída lateral) que permita a la persona mayor desarrollar y asimilar una técnica eficaz, no lesiva, de caer y levantarse del suelo. Sobre esta base, nos centramos en la propuesta metodológica establecida $^{14}$, la cual concentra nuestra atención en el aspecto referido a diseños de deporte e integración social a través de la utilización de actividades deportivas que son modificadas en función de las características de los destinatarios. Para llevar a cabo la enseñanza de los ukemis en las personas mayores planteamos actividades ${ }^{15}$ de asimilación y asimilación asistida vinculadas a los elementos de seguridad necesarios que eviten lesiones y nos permitan automatizar los gestos técnicos de la manera más efectiva. Como consecuencia de este proceso de aprendizaje, se dotará a las personas mayores de las habilidades que le permitan reaccionar de forma refleja ante una posible caída.

El programa tendrá una duración de 2 meses, comenzando el día 7 de abril y finalizando el 7 de junio de 2015. Se realizarán 2 sesiones a la semana. Estas sesiones tendrán una duración de 60 minutos. Todas las sesiones serán grabadas en vídeo para su posterior estudio y análisis. 


\section{Evaluación}

En esta propuesta de innovación se plantean las siguientes evaluaciones:

- Evaluación inicial: Escala Rasgo de fragilidad ${ }^{11,12}$. Calculo del índice de masa corporal a través de la talla (medida con tallímetro) y el peso (con una báscula de baño). Cuestionario de la OMS, para el estudio de caídas en los ancianos (OMS, 1989 ), cuestionario International Falls Efficacy Scale ${ }^{16}$ y El Cuestionario de Salud (SF-36). Así mismo, se evaluarán variables para medir la condición física; Chair stand test, 8 feet up and go test, chair sit and neack, back soath test.

- Evaluación por sesión: a través de lista de asistencia para medir la colaboración y seguimiento de satisfacción de las sesiones con una escala del 1 al 10 al finalizar la sesión.

- Evaluación final. Post-test del Cuestionario de la OMS, para el estudio de caídas en los ancianos (OMS, 198 9), del cuestionario International Falls Efficacy Scale ${ }^{16}$ y del Cuestionario de Salud (SF-36). Se volverá a medir la condición física; Chair stand test, 8 feet up and go test, chair sit and neack, back soath test.

\section{Propuesta de actividades}

Para la intervención sobre el grupo de personas mayores se plantean dos secuencias de aprendizaje. Una asistida autónoma con implementos y otra asistida colaborativa con compañero (con o sin implementos).

En la figura 1 se presenta la progresión de aprendizaje de yoko-ukemi de forma asistida autónoma con implementos (ej.: cinturón, gomas, picas,...). 


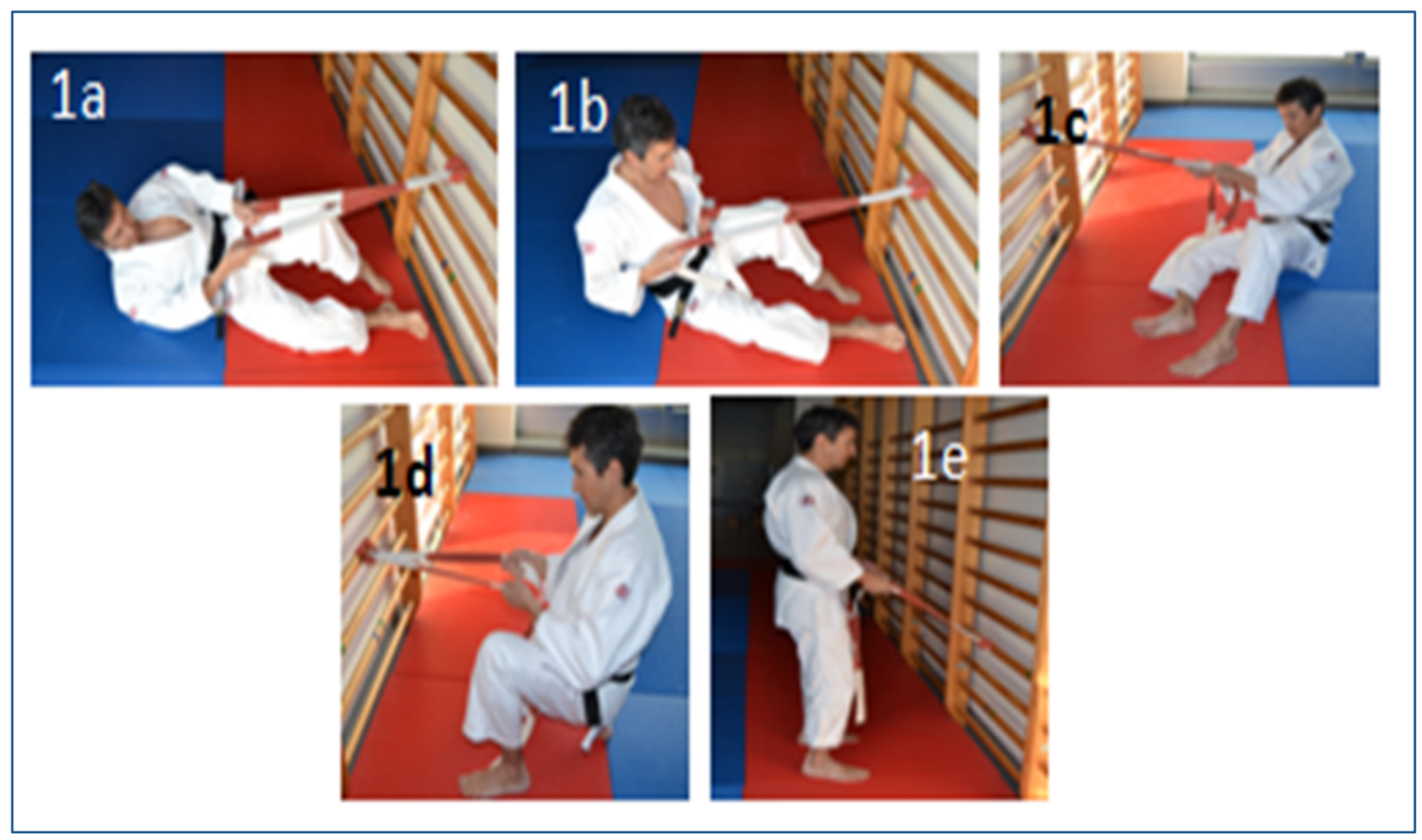

Fig.1 Yoko-ukemi asistido autónomo con implementos

La posición de partida será decúbito supino-lateral derecho (1a). El practicante sujeta el implemento con las manos y, apoyándose en él, realiza flexión lateral de cuello dirigiendo la mirada hacia el pie derecho. Este ejercicio tiene el objetivo de asimilar la posición final de la caída protegiendo la nuca del impacto frente a una caída lateral. Sobre la disposición material del ejercicio 1, y desde la posición final del mismo, contracción abdominal oblicua levantando la zona dorsal del suelo ayudándose de la tracción del implemento (1b). De este modo se minimiza el impacto al rodar por una superficie curva, no impactando los brazos contra el suelo. Sentado con piernas separadas a la anchura de los hombros (1c). El ejecutante, traccionando del implemento, realizará un desequilibrio sobre su lateral derecho iniciado por el cruce de la pierna derecha sobre la izquierda. Como situación final buscaremos acabar en la posición del ejercicio 2 tras rodar por el lateral derecho, desde la cadera, hasta el hombro. En cuclillas, piernas separadas a la anchura de los hombros y rodilla derecha apoyada en el suelo (1d). Sujetando el implemento con las manos, el ejecutante, realizará un desequilibrio sobre su lateral derecho. Una vez apoyada la cadera en el suelo por descenso del centro de gravedad, como situación final buscaremos acabar en la posición del ejercicio 3. Posición de pie 
con piernas abiertas a la altura de los hombros (1e). Implemento sujeto por las manos. Flexión de piernas hasta llegar a la posición del ejercicio 4, lo que permite disminuir la altura de la caída. A continuación, enlazar los ejercicios anteriores en la secuencia 3, 2 y, por último, 1.

En relación al aprendizaje de las caídas laterales (yoko-ukemi) de forma asistida colaborativa con compañero sin implementos planteamos la siguiente secuencia didáctica (ver fig.2). Este ukemi se realizará tanto por el lado derecho, como por el izquierdo.

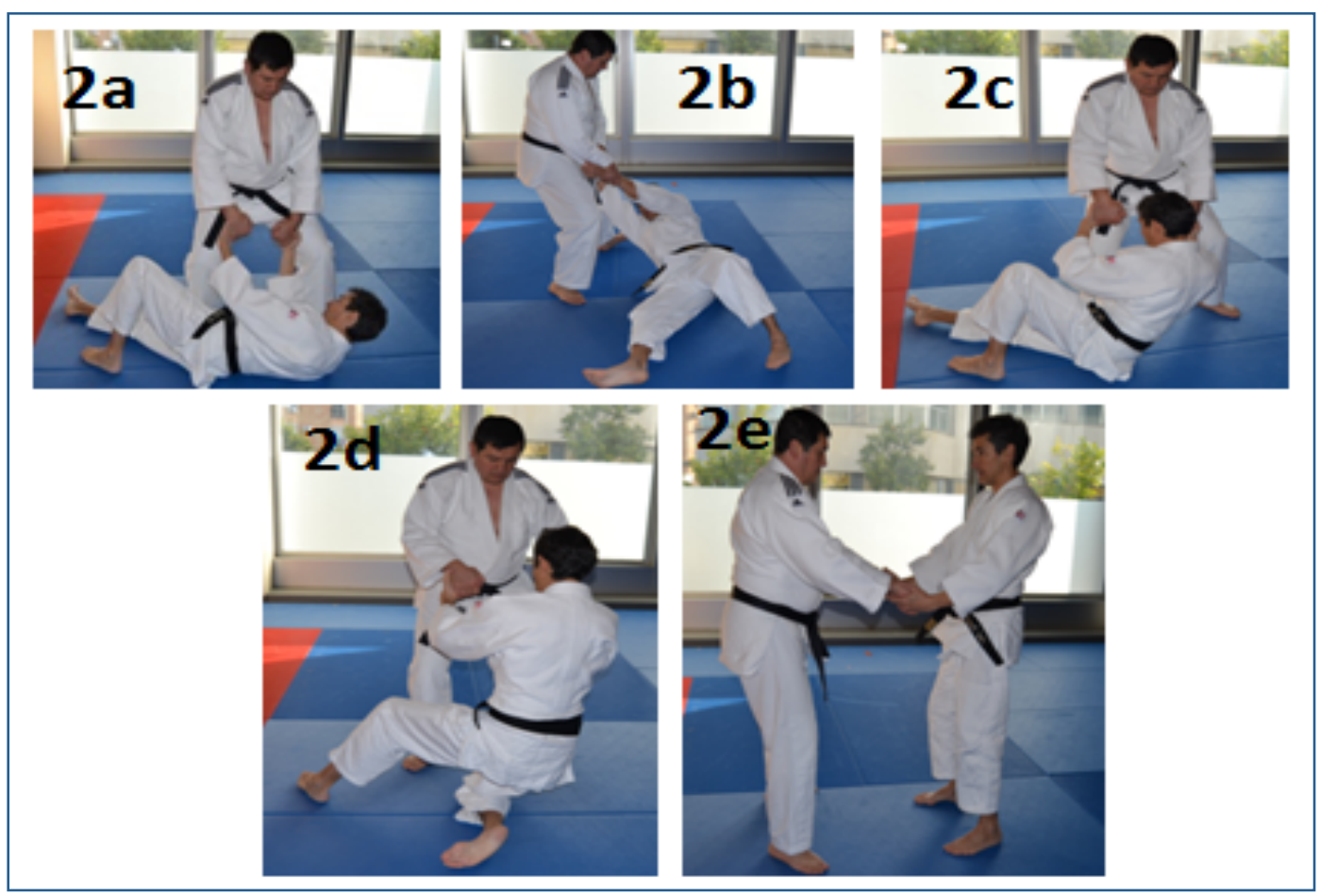

Fig.2 Yoko-ukemi asistido colaborativo con compañero, sin implementos

Comenzamos la progresión en decúbito supino-lateral derecho (2a). El practicante sujeta a su ayudante con las manos y, apoyándose en él, realiza flexión lateral de cuello dirigiendo la mirada hacia el pie derecho. Este ejercicio tiene el objetivo de asimilar la posición final de la caída protegiendo la nuca del impacto frente a una caída lateral. Sobre la disposición material del ejercicio 1 , y desde la posición final del mismo, contracción abdominal oblicua levantando la zona dorsal del suelo ayudándose de la tracción del compañero (2b). De \begin{tabular}{l|l}
-8 & EDUCAJUDO: INNOVACIÓN EDUCATIVA Y DE SALUD EN MAYORES. \\
& APRENDIENDO A CAERSE (YOKO-UKEMI)
\end{tabular} 
este modo se minimiza el impacto al rodar por una superficie curva, no impactando los brazos contra el suelo. Sentado con piernas separadas a la anchura de los hombros (2c). El ejecutante, traccionando de su ayudante, realizará un desequilibrio sobre su lateral derecho iniciado por el cruce de la pierna derecha sobre la izquierda. Como situación final buscaremos acabar en la posición del ejercicio 2 tras rodar por el lateral derecho, desde la cadera, hasta el hombro. En cuclillas, piernas separadas a la anchura de los hombros y rodilla derecha apoyada en el suelo $(2 \mathrm{~d})$. Sujetando su ayuda con las manos, el ejecutante, realizará un desequilibrio sobre su lateral derecho. Una vez apoyada la cadera en el suelo por descenso del centro de gravedad, como situación final buscaremos acabar en la posición del ejercicio 3. Posición de pie con piernas abiertas a la altura de los hombros (2e). El sujeto colaborador ayuda por las manos. Flexión de piernas hasta llegar a la posición del ejercicio 4, lo que permite disminuir la altura de la caída. A continuación, enlazar los ejercicios anteriores en la secuencia 3, 2 y, por último, 1.

\section{CONCLUSIONES}

Una vez realizada la intervención y recogidos y analizados los resultados esperamos una gran repercusión de esta propuesta para la salud de las personas mayores. Se espera alcanzar una utilidad máxima ya que tendremos una herramienta para reducir el número de caídas de las personas mayores, el daño producido en las mismas, además de otros beneficios adheridos al mismo como la mejora del equilibrio, la marcha, la coordinación, la atención, la memoria y la capacidad funcional. Conllevan una mejora de la autoestima, la confianza y es una medida activa de la prevención de las caídas. 


\section{REFERENCIAS}

1. INE. Proyección de la Población de España a Corto plazo, 2013-2023. Instituto Nacional de Estadística. España; 2013;1-9.

2. 2. Europeia C. Demography report 2010: Older, more numerous and diverse Europeans. Comission Staff Working Document. Luxembourg: EUROSTAT. 2011.

3. Fried LP, Tangen CM, Walston J, Newman AB, Hirsch C, Gottdiener J, et al. Frailty in older adults: Evidence for a phenotype. Journals Gerontol - Ser A Biol Sci Med Sci. 2001;56(3).

4. Andradas E, Labrador MV, Lizarbe V, Molina M. Documento de consenso sobre prevención de fragilidad y caídas en la persona mayor. Estrategia de Promoción de la Salud y Prevención en el SNS. Misterio d. Misterio de Sanidad SS e I, editor. España: Misterio de Sanidad, Servicios Sociales e Igualdad; 2014.

5. Aparicio VA, Carbonell-Baeza A, Delgado-Fernández M. Beneficios de la actividad física en personas mayores. Rev Int Med y Ciencias la Act Fis y del Deport. 2010;10(40):556-76.

6. Causapié P. Envejecimiento activo. Libro blanco. (España) I de M y SS, editor. españa; 2011.

7. Riocerezo L. El envejecimiento saludable: actividad física en la prevención de caídas del anciano. 2014;

8. Pocecco E, Gatterer H, Ruedl G, Burtscher M. Specific exercise testing in judo athletes. Arch Budo. 2012;8(3):133-9.

9. Montero C, López JL. Impacto producido por la técnica seoiotoshi. Relación con años de práctica y grado en judo. Rev Artes Marciales Asiáticas. 2014;9(1):32-41.

10. Pocecco E, Ruedl G, Stankovic N, Sterkowicz S, Del Vecchio FB, GutiérrezGarcía C, et al. Injuries in judo: a systematic literature review including suggestions for prevention. Br J Sports Med. diciembre de 2013;47(18):113943.

11. García-García FJ, Carcaillon L, Fernandez-Tresguerres J, Alfaro A, Larrion JL, Castillo $C$, et al. A new operational definition of frailty: the Frailty Trait Scale. J Am Med Dir Assoc. Elsevier Inc.; mayo de 2014;15(5):371.e7-371.e13.

12. García-García FJ, Larrión JL, Rodríguez L. Frailty: a phenotype under review. Gac Sanit. diciembre de 2011;25 Suppl 2(SUPPL. 2):51-8.

13. Taira S, Suárez JJ, Montero M. La esencia del judo. Satori; 2014.

14. Marta C, Gaspar M. Deporte, actividad fisica e inclusión social: Una guía para la intervención social a través de las actividades deportivas. España: Consejo Superior de Deportes; 2010. 198 p.

15. Sánchez MLZ. La didáctica de la Educación Física. Pearson Educación; 2002.

16. Yardley L, Beyer N, Hauer K, Kempen G, Piot-Ziegler C, Todd C. Development and initial validation of the Falls Efficacy Scale-International (FES-I). Age Ageing. Br Geriatrics Soc; 2005;34(6):614-9.

Referencias totales citadas: 16

Referencias citadas correspondientes a la Rev Ib CC Act Fís Dep: 0 